\title{
PLASMON RESONANCES OF SPHERICAL AND ELLIPSOIDAL NANOPARTICLES
}

\section{J.-W. Liaw}

Materials Research Laboratories

Industrial Technology Research Institute

Hsinchu, Taiwan, 310, ROC

\section{K. Kuo and C. N. Liao}

Institute of Applied Mechanics, National Taiwan University Taipei, Taiwan, 106, ROC

Abstract-Surface plasmon resonance (SPR) of a single metallic nanoparticle is analyzed and simulated via multi-multipole method for 3D problems. The excitation, at optical frequencies, of the SPR leads to an extremely strong field in the vicinity of the nanoparticle. Numerical results indicate that a red shift of SPR is induced for an ellipsoidal nanoparticle, compared to a spherical one. Two structures of core-shelled spherical nanoparticles are also studied; one is a nanosphere of $\mathrm{Ag}$ shell with an oxide core, and the other one is $\mathrm{Ag}$ core with an oxide shell $\left(\mathrm{ZrO}_{2}\right.$ or $\left.\mathrm{SiO}_{2}\right)$. Numerical results illustrate the SPR of these two core-shelled structures are quite different from each other and different from that of a solid one. It suggests that one can manipulate the optical response on demand by tuning the core/shell ratio and the permittivity of shell or core.

\section{INTRODUCTION}

Optical properties of plasmon resonant metallic nanoparticles are of great interest owing to the ability of controlling optical fields on the nanometer scale. The phenomenon can be utilized to manipulate the light for versatile applications, e.g., nanosensors [1], photocatalyst [2] etc. Recently, non-spherical nanoparticles as well as core-shelled nanoparticles have attracted lots of attention, since they provides new varieties to tune the optical properties [3] by changing not only the shape, size and material of the core, but also the thickness and the 
material dielectric of the shell. There are two main structures of the core-shelled nanoparticles having been synthesized in the last decade by chemists, namely $\mathrm{Au}$ shell with a silica core $\left(\mathrm{SiO}_{2}\right)$ [4] and $\mathrm{Au}$ or $\mathrm{Ag}$ core with an oxide shell (e.g., $\mathrm{ZrO}_{2}$ or $\mathrm{TiO}_{2}$ ) [5]. In the paper, the effects of ellipsoidal shapes and of the core/shell ratio on the plasmon resonance of the above-mentioned nanostructures will be studied by using muti-multipole expansion (MMP).

\section{THEORY}

MMP was originally developed for systems with piecewise homogeneous, isotropic, and linear media [6,7] and has been applied successfully for computing electromagnetic field in many applications. According to the MMP proposed by Hafner [7], the electric and magnetic fields can be expanded with respect to several centers, and each series can then be truncated to finite terms. Thus, the electric and magnetic fields, in the exterior region of all scatterers, can be expressed as:

$$
\left\{\begin{array}{l}
\mathbf{E}=\sum_{\alpha=1}^{q} \sum_{n=1}^{N} \sum_{m=-n}^{n}\left(a_{m n, \alpha} \mathbf{M}_{m n, \alpha}+b_{m n, \alpha} \mathbf{N}_{m n, \alpha}\right) \\
\mathbf{H}=\frac{k}{j \omega \mu} \sum_{\alpha=1}^{q} \sum_{n=1}^{N} \sum_{m=-n}^{n}\left(a_{m n, \alpha} \mathbf{N}_{m n, \alpha}+b_{m n, \alpha} \mathbf{M}_{m n, \alpha}\right)
\end{array}\right.
$$

where $q=\ell \times p$ is the total number of the expanding centers, and $\ell$ and $q$ are the numbers of the scatterers and the numbers of the centers per each scatterer, respectively. For the fields in the interior region of the any one of scatterer, the expressions are exactly the same as (1), except that $q=p$, i.e., only the centers of the particular scatterer are needed in the expansion of interior fields, and the bases functions $\mathbf{M}_{m n}$ and $\mathbf{N}_{m n}$ should be replaced by the appropriate ones. Coefficients $a$ and $b$ have to be determined to accommodate the incident fields and boundary conditions of scatterers. Basis functions $\mathbf{M}$ and $\mathbf{N}$ are defined as

$$
\left\{\begin{aligned}
\mathbf{M}_{m n} & =\nabla \times\left(\psi_{m n} \mathbf{e}_{R}\right) \\
\mathbf{N}_{m n} & =\frac{1}{k} \nabla \times \nabla \times\left(\psi_{m n} \mathbf{e}_{R}\right)
\end{aligned}\right.
$$

where $\psi_{m n}=z_{n}(k R) P_{n}^{|m|}(\cos \theta) e^{j m \phi}$ satisfy homogeneous scalar Helmholtz equation in spherical coordinate, $z_{n}$ are the appropriate spherical Bessel functions, and $P_{n}^{|m|}$ the associated Legendre polynomials, while $m=-n, \ldots, n$, and $n=1,2, \ldots, \infty$. 


\section{DISCUSSION}

Consider a nanoparticle illuminated by TEM waves with the magnetic field polarized in the y-direction and wavelengths ranging from $300 \mathrm{~nm}$ to $500 \mathrm{~nm}$. The accuracy of the numerical code has been verified first by checking for the cases of a solid spherical scatterer against the analytic solution from Mie theory. The relative errors are smaller than $0.01 \%$. Experimental data of permittivity of silver obtained by Johnson and Christy [8] will be used in numerical examples throughout the article unless mentioned explicitly.

Scattering cross-sections (SCS) for the cases of an ellipsoidal nanoparticle of $10 \mathrm{~nm} \times 10 \mathrm{~nm} \times 20 \mathrm{~nm}$ versus wavelengths are plotted in Figure 1 for various incident angels. It is easy to see that there is only one resonant peak in SCS spectra for the cases of incident wave along the major axis (i.e., $\theta=0^{\circ}$ ), and along the minor axis (i.e., $\theta=90^{\circ}$ ) of ellipsoidal nanoparticle, where the resonant wavelengths are around $347 \mathrm{~nm}$ and $412 \mathrm{~nm}$, respectively. Moreover, the maximum of SCS for the latter case is larger than that of the former case. On the other hand, for cases of inclined incident with incident angle $\theta=45^{\circ}$, both resonant wavelengths of $347 \mathrm{~nm}$ and $412 \mathrm{~nm}$ are pronounced. The near-field amplitude distributions of electric fields at the corresponding resonant wavelengths are shown in Figure 2.

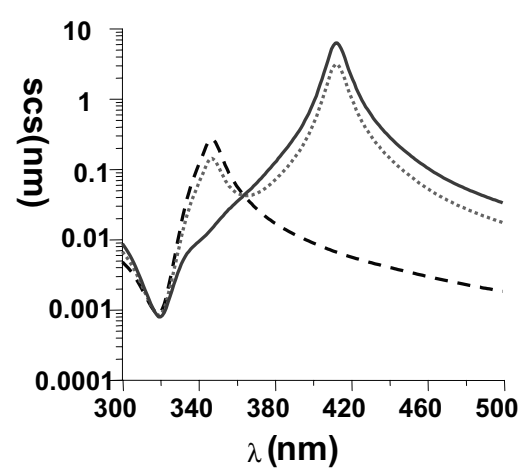

Figure 1. Scattering cross-sections (SCS) versus wavelengths for ellipsoidal nanoparticles of $10 \mathrm{~nm} \times 10 \mathrm{~nm} \times 20 \mathrm{~nm}$ by TEM wave of various incident angles: $\theta=0^{\circ}(--) ; \theta=90^{\circ}(-) ; \theta=45^{\circ}(\cdots \cdots)$.

Scattering cross-sections (SCS) for the cases of a spherical coreshell nanoparticle versus wavelengths are plotted in Figures 3 and 4 for various shell thicknesses. In Figure 3, the nanoparticle is of $\mathrm{Ag}$ core with an oxide shell. The core has a radius of $10 \mathrm{~nm}$, while the shell is made either of $\mathrm{SiO}_{2}$ (with permittivity 2.1) or $\mathrm{ZrO}_{2}$ (with 

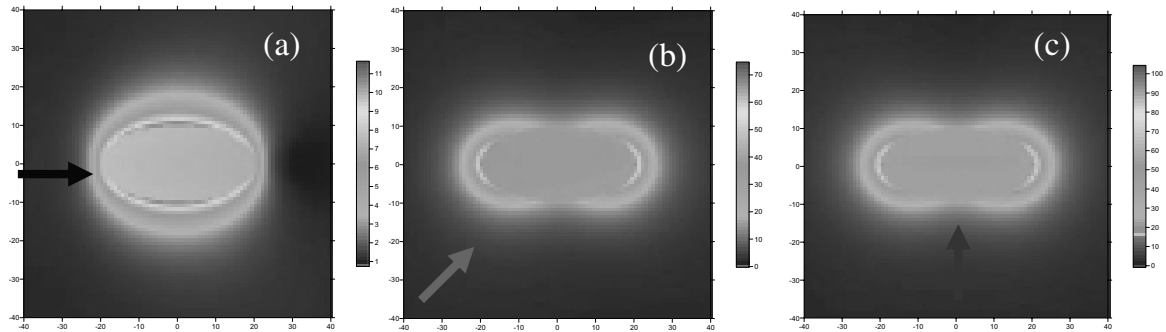

Figure 2. Near-field amplitude distribution of electric field in $x z$-plane at resonant wavelengths of an ellipsoidal nanoparticle of $10 \mathrm{~nm} \times 10 \mathrm{~nm} \times 20 \mathrm{~nm}$ illuminated by TEM waves with various incident angles: (a) $\theta=0^{\circ}, \lambda=347 \mathrm{~nm}$ (b) $\theta=45^{\circ}, \lambda=412 \mathrm{~nm}$ (c) $\theta=90^{\circ}, \lambda=412 \mathrm{~nm}$.
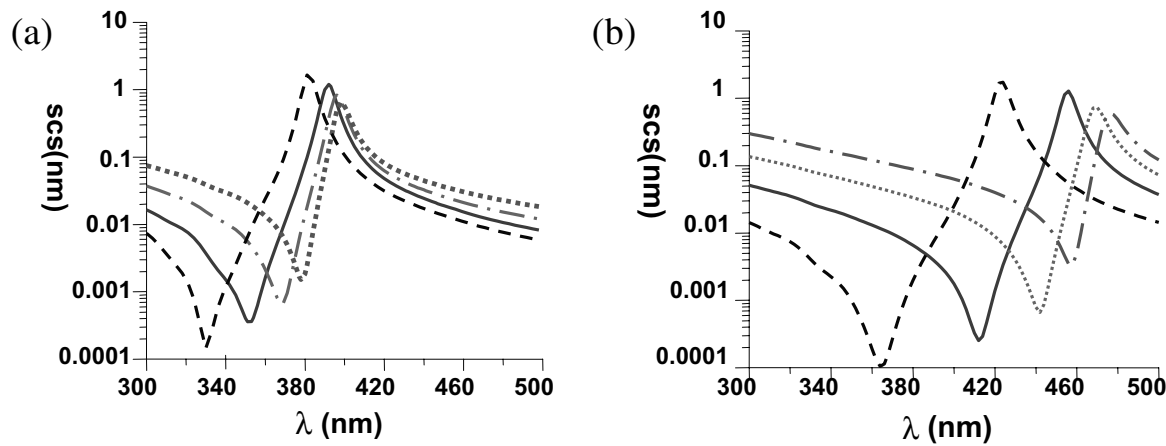

Figure 3. Scattering cross-sections (SCS) versus wavelengths for a spherical core-shell nanoparticle with $\mathrm{Ag}$ core of radius $R=10 \mathrm{~nm}$ and an oxide shell. The shell is (a) $\mathrm{SiO}_{2}, \varepsilon_{r}=2.1$ (b) $\mathrm{ZrO}_{2} \varepsilon_{r}=4.2$ of various shell-thickness: $5 \mathrm{~nm}(--) ; 10 \mathrm{~nm}(-)$; $15 \mathrm{~nm}(\cdots \cdots)$; and $20 \mathrm{~nm}(-\cdot)$.

permittivity 4.2 ) with various thicknesses. It is easy to see that there is only one resonant peak in SCS spectra for each of these cases. The peaks occur at wavelengths around 380-400 nm for cases of $\mathrm{SiO}_{2}$ shell, while at wavelengths larger than $420 \mathrm{~nm}$ for cases of $\mathrm{ZrO}_{2}$ shell. It is also clear from the figure that the thicker the shell, the more the red-shift of the peak. Moreover, by comparing subfigures (a) and (b), for a fixed shell's thickness, the red shifts of SPR of Ag core covered by $\mathrm{ZrO}_{2}$ are always more severe than those covered by $\mathrm{SiO}_{2}$. These results are consistent with a number of experiments $[9,10]$; where the peak position of SPR of a single metallic nanoparticle exhibited a red 
shift when nanoparticle embedded in a host with higher permittivity compared to those in a lower-permittivity host, since the shell acts as a part of host to the Ag core. Moreover, the role of shell as a host to the core will be more profound as the thickness of the shell getting thicker and thicker. This provides a reasonable explanation to a direct observation from subfigures (a) and (b) that the thicker the shell, the more the red shift.
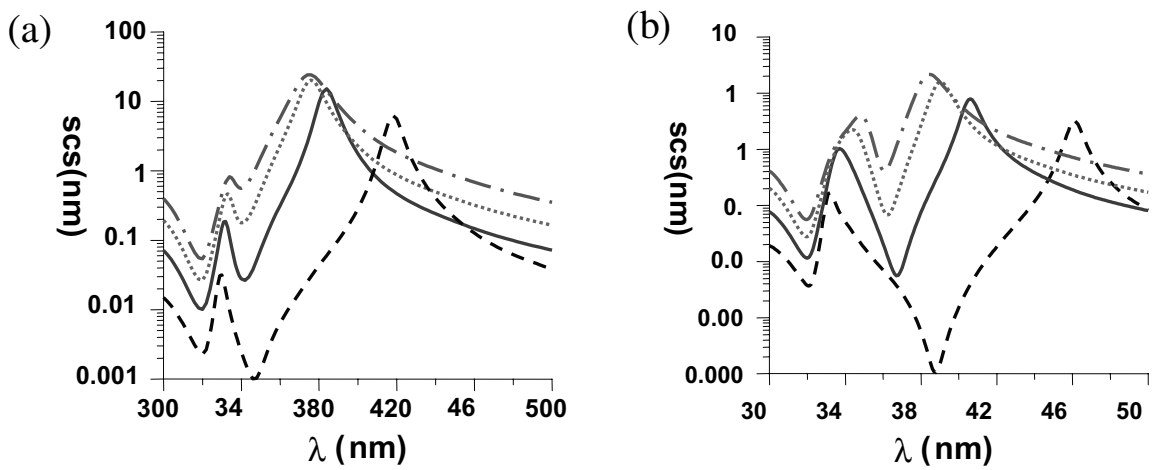

Figure 4. Scattering cross-sections (SCS) versus wavelengths for a spherical core-shell nanoparticle with $\mathrm{Ag}$ shell of various thicknesses with a core of radius $R=10 \mathrm{~nm}$ made of (a) $\mathrm{SiO}_{2}, \varepsilon_{r}=2.1$ (b) $\mathrm{ZrO}_{2}, \varepsilon_{\mathrm{r}}=4.2$. The thicknesses of shells are $5 \mathrm{~nm}(--) ; 10 \mathrm{~nm}(-)$; $15 \mathrm{~nm}(\cdots \cdots)$; and $20 \mathrm{~nm}(-\cdot)$.

Figure 4 are the SCS spectra of Ag shell of various thicknesses with a silica core $\left(\mathrm{SiO}_{2}\right)$ or zirconia core $\left(\mathrm{ZrO}_{2}\right)$ of radius $R=10 \mathrm{~nm}$. There are two resonant peaks in SCS spectra for each of these cases to illustrate the SPR of these core-shell structures are more complicated than their counterparts in Figure 3. It can also be seen from Figure 4 that when the thickness of the Ag shell becomes thinner, the first peak shifts to a longer wavelength (a red shift) and the second peak to a shorter wavelength (a blue shift). This can be served as an additional tuning factor for a desirable optical property. By comparing subfigures (a) and (b), it is clear that the higher the permittivity of the core is (i.e., subfigure b), the more shifting the two peaks are. Furthermore, when the thickness of the Ag shell becomes larger, the two peaks are getting closer to each other to even have the tendency to merge together. This might be attributed to the fact that the configuration of a thick metallic shell is similar to a solid metallic nanoparticle; i.e., the effects of the core-shell structure become insignificant.

The above-mentioned cases of $\mathrm{ZrO}_{2}$ core with $\mathrm{Ag}$ shell are 


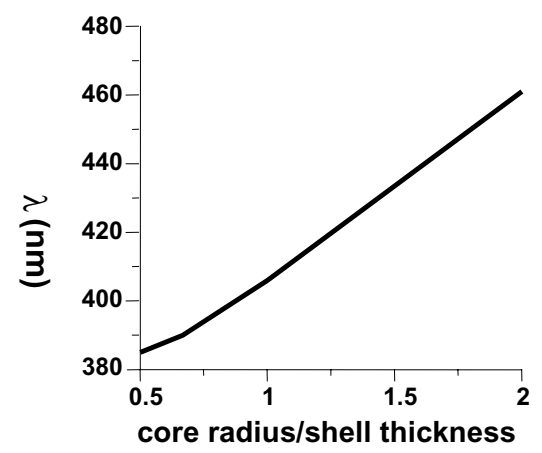

Figure 5. Resonant wavelength versus radius-thickness ratio for a spherical core-shell nanoparticle of $\mathrm{Ag}$ shell with a core of $\mathrm{ZrO}_{2}$.

considered in Figure 5 further. The first peak (the longer resonant wavelength) of SPR versus the core radius to shell thickness ratio are plotted in Figure 5. The relation is almost linear. This result could be useful toward to tune the resonance wavelength by adjusting the radius-thickness ratio.

Finally, it is also worthwhile to compare the hybridization model proposed by Prodan et al. [11] to describe the surface plasmon in the nanoshell. According to Prodan, the plasmon resonance in the nanoshell can be explained by the interaction of plasmon resonance in the nano-sphere and nano-cavity. The relative permittivity of core is set to 1, i.e., vacuum, and Drude's model is used to describe the dispersion of silver shell's permittivity in order to check against Prodan's result. The two resonant wavelengths are then found from numerical values of SCS spectra as $168 \mathrm{~nm}$ and $296 \mathrm{~nm}$ for the inner and outer radii being $10 \mathrm{~nm}$ and $20 \mathrm{~nm}$. It is very close to the values predicted by hybridization model which are $166 \mathrm{~nm}$ and $278 \mathrm{~nm}$, respectively.

\section{CONCLUSIONS}

In the paper, several factors, e.g., the aspect ratio, the core/shelled ratio and the core/shell materials, which can control the SPR of a single metallic nanoparticle, were studied by MMP. Scattering cross-sections (SCS) versus wavelengths as well as the amplitude distributions of electric near-fields at some resonant wavelengths were presented.

For the cases of ellipsoidal nanoparticles, there was only one, but different, resonant peak in SCS spectra for the cases of incident wave along the major and the minor axes. On the other hand, both two 
resonant peaks were excited for the case of inclined angle of incidence.

For the cases of a spherical core-shell nanoparticle of $\mathrm{Ag}$ core with an oxide shell, the results showed that the thicker the shell, the more the red-shift of the SPR peak. And the red shifts of SPR of core-shell with $\mathrm{ZrO}_{2}$ shell were more severe than those with $\mathrm{SiO}_{2}$ shell. On the other hand, for the cases of $\mathrm{Ag}$ shell with core of $\mathrm{SiO}_{2}$ and $\mathrm{ZrO}_{2}$, there were two resonant peaks for each case. And as the thickness of the Ag shell became thinner, the first peak shifted to a longer wavelength (a red shift) and the second peak to a shorter wavelength (a blue shift). Again, the shifting of two SPR peaks for $\mathrm{ZrO}_{2}$ core was more evident than those of $\mathrm{SiO}_{2}$ core.

The effect of the aspect ratio of a core-shelled ellipsoidal nanoparticle on SPR is expected to be even more significant. The topic is currently being conducted by using MMP now.

\section{ACKNOWLEDGMENT}

Part of this work was carried out in the course of research sponsored by the Ministry of Economic Affairs through Industrial Technology Research Institute in Taiwan, Republic of China (Grant No. A321XS9L10). Thanks are due to Dr.J. K. Wang of Center for Condensed Matter Sciences, National Taiwan University, for very helpful discussions and suggestions.

\section{REFERENCES}

1. Prodan, E., P. Nordlander, and N. J. Halas, Nano Letters, Vol. 3, 1411, 2003.

2. Grady, N. K., N. J. Halas, and P. Nordlander, Chemical Phys. Lett., Vol. 399, 167, 2004.

3. Hirakawa, T. and P. V. Kamat, Langmuir, Vol. 20, 5645, 2004.

4. Oldenburg, S. J., J. B. Jackson, S. L. Westcott, and N. J. Halas, Appl. Phys. Lett., Vol. 75, 2879, 1999.

5. Tom, R. T., A. S. Nair, N. Singh, M. Aslam, C. L. Nagendra, R. Philip, K. Vijayamohanan, and T. Pradeep, Langmuir, Vol. 19, 3439, 2003.

6. Moreno, E., D. Eni, C. Hafner, and R. Vahldieck, J. Opt. Soc. Am. A., Vol. 19, 101, 2002.

7. Hafner, C., The Generalized Multipole Technique for Computational Electromagnetics, Artech. House, Boston, 1991.

8. Johnson, B. and R. W. Christy, Phy. Rev. B., Vol. 6, 4370, 1972.

9. Link, S., et al., J. Phys. Chem. B, Vol. 103, 3073, 1999. 
10. Liu, Z., et al., Appl. Phys. Lett., Vol. 72, 1823, 1998.

11. Prodan, E., C. Radloff, N. J. Halas, and P. Nordlander, Science, Vol. 302, 419, 2003. 